\title{
Stability and auctions in labor markets with job security
}

Article · May 2017

DOI: 10.1016/j.econlet.2017.02.024

\section{CITATIONS}

0

4 authors, including:

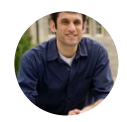

Robert D. Kleinberg

Cornell University

129 PUBLICATIONS 4,096 CITATIONS

SEE PROFILE

Rann Smorodinsky

Technion - Israel Institute of Technology

44 PUBLICATIONS 652 CITATIONS

SEE PROFILE
READS

8

\section{Ron Lavi}

Technion - Israel Institute of Technology

48 PUBLICATIONS $\quad \mathbf{1 , 2 5 9}$ CITATIONS

SEE PROFILE

All content following this page was uploaded by Rann Smorodinsky on 15 May 2017.

The user has requested enhancement of the downloaded file. All in-text references underlined in blue are added to the original document and are linked to publications on ResearchGate, letting you access and read them immediately. 


\title{
Stability and Auctions in Labor Markets with Job Security*
}

\author{
$\mathrm{Hu} \mathrm{Fu}^{\dagger} \quad \underline{\text { Robert Kleinberg }}^{\ddagger} \quad \underline{\text { Ron Lavi }}^{\S} \quad \underline{\text { Rann Smorodinsky }}{ }^{\Phi}$
}

\begin{abstract}
JS-stable outcomes have been introduced by Fu et al. (2016) as a stability concept for labor markets in the Kelso-Crawford framework with the addition of job security regulation. In this note we show that any assignment of workers to firms in such an outcome must be a Nash equilibrium outcome of a natural auction where firms compete for workers. This parallels results in the literature about stable outcomes and similar auctions, and yields price-of-anarchy bounds for such auction games.
\end{abstract}

JEL Classification Numbers: C78, D44, D82

Keywords: Matching, Stability, Labor market, Job security, Auction.

*The results in this note were presented in the 13th ACM Conference on Electronic Commerce (EC'12), see Fu et al. (2012). Only the abstract (25 lines) was included in the proceedings.

UUniversity of British Columbia, Department of Computer Science. Email: fu.hu.thu@gmail.com. This work was done while the author was a PhD student in the Computer Science Department at Cornell University and a postdoc at Microsoft Research New England and California Institute of Technology.

${ }^{\ddagger}$ Computer Science Department, Cornell University. Email: rdk@cs.cornell.edu. Portions of this note were written while the author was a researcher at Microsoft Research New England.

${ }^{\S}$ Faculty of Industrial Engineering and Management, Technion - Israel Institute of Technology. Email: ronlavi@ie.technion.ac.il. This author is supported by a Marie-Curie IOF fellowship.

TFaculty of Industrial Engineering and Management, Technion - Israel Institute of Technology. Email: rann@ie.technion.ac.il. Smorodinsky gratefully acknowledges the support of ISF grant 2016301, the joint MicrosoftTechnion e-Commerce Lab, Technion VPR grants, the Bernard M. Gordon Center for Systems Engineering at the Technion and the Technion Autonomous System Program at the Technion. 


\section{Introduction}

The purpose of this note is to study a connection between a model of labor markets with regulation recently defined and analyzed in Fu et al. (2016) and a model of simultaneous single-item auctions which was originally suggested by Bikhchandani (1999) and which has received significant attention in the last few years in the computer science literature on algorithmic game theory. This connection yields new results in both models. In particular, the results of Fu et al. (2016) on labor markets with regulation imply (via the connection we establish here) new bounds on the price of anarchy and the price of stability of simultaneous second price auctions as well as new guarantees on the existence of pure Nash equilibria in this auction game.

In the classical labor market model due to Kelso and Crawford (1982) there is a set of firms $N$, a set of workers $M$, and a production function $v^{n}: 2^{M} \rightarrow \Re_{+}$for every firm $n \in N$, where $v^{n}(S)$ is the production value of firm $n$ if it hires a subset workers $S \in 2^{M}$. Three main results of this theory are that gross-substitutability of all production functions is a sufficient condition for the existence of a stable matching of workers and firms, that all stable matchings are efficient, and that in fact gross-substitutability is also a necessary condition for the above two properties to hold (this last property is due to Gul and Stacchetti (1999)).

Bikhchandani (1999) studies a complete-information auction game where firms compete simultaneously for employees by proposing salaries, and employees pursue the highest offer. This is known in the literature on combinatorial auctions as a First Price Item Bidding (FPIB) auction. Bikhchandani (1999) shows a connection between this auction game and the classic labor market model described above, by showing a correspondence between the set of Nash equilibrium (NE) outcomes of the FPIB game and the set of stable outcomes of a labor market ${ }^{1}$

In a recent paper (Fu et al., 2016) we modify the classic labor market model and study labor markets with regulation designed to provide employees with job security. To capture job security we have introduced a weaker solution concept termed JS-stability (where JS stands for job security). That previous paper has three main results: it provides sufficient conditions on the structure of the production functions that ensure the existence of JS-stability, it shows that the welfare in any JS-stable outcome is at least half of the optimal welfare, and it describes necessary and sufficient conditions for the existence of efficient JS-stable outcomes. These results therefore provide a mirror image of the three main results of classic labor markets (without job security) described above.

Continuing this thread of thought, our result in this current note parallels the connection between the classic labor market and the FPIB game of Bikhchandani (1999). Specifically, we show a connection between labor markets with job security and the Second Price Item Bidding (SPIB) auction game, introduced in (Christodoulou et al. 2008). The difference between FPIB and SPIB is that in the latter an employee's salary is determined by the second highest offer and not the first. While Bikhchandani (1999) shows a correspondence between the set of pure NE outcomes of the FPIB auction game and the set of stable matchings of the classic labor market (without regulation), we show here a correspondence between the set of pure NE outcomes of the SPIB auction game and the set of JS-stable outcomes in our model of labor markets with regulation. The two theories of labor markets with and without job security are therefore parallel with respect to this property as well. As mentioned above, as an immediate corollary of this connection we obtain several results regarding existence of pure Nash equilibria in the SPIB auction game, and

\footnotetext{
${ }^{1}$ The terminology of Bikhchandani (1999) uses buyers and items instead of firms and workers, but the difference is only semantic, as we explain below.
} 
the price of anarchy and price of stability in this game.

Section 2 gives more details on the model of a labor market with regulation. Section 3 analyzes the connection between the labor market model and the auction model, and describes some corollaries that this connection yields.

\section{Labor Markets with Regulation}

The classical labor market model due to Kelso and Crawford (1982) is given by a tuple $\left(N, M,\left(v^{n}\right)_{n \in N}\right)$, where $N$ denotes the set of firms, $M$ the set of workers and $v^{n}: 2^{M} \rightarrow \Re_{+}$is the production function of firm $n$ in monetary units. We assume throughout that the functions $v^{n}$ are monotonically increasing and calibrate $v^{n}(\emptyset)=0$. A job market allocation is a pair $(A, s)$, consisting of an assignment $A=\left\{A^{1}, \cdots, A^{N}\right\}$ and a salary vector $s=\left\{s_{m}\right\}_{m \in M}$. An assignment $A$ is a partition of the set $N$ of workers, where $A^{n}$ is the set of workers employed by firm $n$. Given an allocation $(A, s)$ the utility of worker $m$ is her salary $s_{m}$ and the utility of firm $n$ is $\Pi^{n}(A, s)=v^{n}\left(A^{n}\right)-\sum_{m \in A^{n}} s_{m}$, i.e., the value that the firm obtains from employing the workers in $A^{n}$ minus the sum of salaries to these workers. The efficiency level (or welfare level) of an assignment $A$ is $P_{v}(A)=\sum_{n} v^{n}\left(A^{n}\right) 2^{2}$ The central solution concept in the literature on labor markets is that of stability:

Definition 1. An allocation $(A, s)$ is individually rational (IR) if, for all $n, \Pi^{n}(A ; s) \geq 0$ and $s_{m} \geq 0$ for all $m \in A^{n}$.

Definition 2. A coalition $\{n, C\}$ is a blocking coalition for an allocation $(A, s)$ if and only if there exists a vector of salaries, $\hat{s} \in \Re_{+}^{C}$, such that:

1. $\hat{s}_{m} \geq s_{m} \quad \forall k \in N, m \in A^{k} \cap C$ (workers in $C$ are better-off),

2. $v^{n}(C)-\sum_{m \in C} \hat{s}_{m} \geq v^{n}\left(A^{n}\right)-\sum_{m \in A^{n}} s_{m}$ (firm $n$ is better-off),

with at least one of the inequalities being strict. An allocation $(A, s)$ is stable if and only if it is IR and there exist no blocking coalitions for it.

Stable outcomes (whenever they exist) can be shown to exhibit maximum efficiency level. However, they are guaranteed to exist only when firms' production functions are gross substitutes (Gul and Stacchetti, 1999). Partly motivated by an attempt to broaden existence of stable outcomes and partly motivated by job security regulations common in many labor markets, the current set of authors considered in Fu et al. (2016) a weaker notion of stability termed JS-stability, where JS stands for Job Security:

Definition 3. A coalition $\{n, C\}$ is a JS-blocking coalition for an allocation $(A, s)$ if and only if it is a blocking coalition, and additionally $A^{n} \subset C$. An allocation $(A, s)$ is JS-stable if and only if it is IR and there exist no JS-blocking coalitions for it.

The extra requirement $A^{n} \subset C$ is what captures job security. In other words, a blocking firm can only consider adding new workers and cannot unilaterally dismiss any of its current workers. A detailed discussion of the advantages and disadvantages of this stability concept is given in our previous paper (Fu et al., 2016).

\footnotetext{
${ }^{2}$ This model can be easily extended to allow for employees' utility to be firm-dependent and to allow for unemployed workers. For more details the reader is referred to Fu et al. $(2016)$.
} 
In Fu et al. (2016) we showed that JS-stable outcomes are guaranteed to exist for a class of valuation functions much larger than gross-substitutes. The efficiency loss when relaxing the stability notion to JS-stability was quantified as follows.

Definition 4. A firm's production function $v$ is fractionally subadditive on a set $C \subseteq M$, if there exist salaries $s \in \Re_{+}^{C}$ such that $\sum_{m \in C} s_{m}=v(C)$ and $\forall D \subset C, \sum_{m \in D} s_{m} \leq v(D)$. A production function $v$ is fractionally subadditive if it is fractionally subadditive on all subsets of $M$.

Definition 5. A firm's production function $v$ is almost fractionally subadditive (AFS) if:

1. For any $C \subset M$ (excluding $C=M) v$ is fractionally subadditive on $C$, and

2. $v(M) \leq \frac{1}{|M|-1} \sum_{m \in M} v(M \backslash m)$.

AFS strictly contains FS (Fu et al. 2016), and FS significantly expands the class of gross-substitutes valuations (Lehmann et al. 2006). The main results in Fu et al. (2016) can be summarized as follows:

Theorem 1 (Fu et al. 2016). Given any job market $\left(N, M,\left(v^{n}\right)_{n \in N}\right)$,

1. If an allocation $(A, s)$ is JS-stable, and if $\bar{A}$ is an assignment that maximizes the efficiency level, then $P(A) \geq \frac{1}{2} P(\bar{A})$.

2. If each $v^{n}$ is in AFS, then an efficient JS-stable outcome is guaranteed to exist.

Furthermore, for any $u \notin A F S$ there exist an integer $k$ and production functions $v^{1}, \ldots, v^{k} \in$ AFS such that no efficient JS-stable outcome exists in the job market with production functions $\left(u, v^{1}, \ldots, v^{k}\right)$.

We refer the reader to Fu et al. (2016) for intuition of the AFS class and the proof of the theorem.

\section{Assigning Workers Through an Auction}

The notion of a stable outcome tells us what we can expect from an outcome in a labor market. Put differently, a non-stable outcome is something we cannot expect, as either one of the players (firms or workers) has negative utility, and in this case it can simply walk away from the market, or alternatively a coalition of a firm and workers can jointly walk away from the market and be better off. However, how markets reach stability is left open. To cope with this question we look at the market as a non-cooperative game where firms bid simultaneously for workers. Each worker is then assigned to work for the highest bidder at a salary equal the second highest bid. We refer to this game as a second-price item-bidding (SPIB) auction.

Nash equilibrium outcomes of this game were first analyzed by Christodoulou et al. (2008). Note that a trivial Nash equilibrium always exists in any SPIB auction, where one bidder bids "infinity" on every item and every other bidder bids zero. Such equilibria are neither interesting nor realistic. To exclude such equilibria, a bidder is said to be no-overbidding if the sum of her bids on any subset of items is no larger than her valuation for the subset. An equilibrium is nooverbidding if all bidders' equilibrium strategies are no-overbidding. Christodoulou et al. (2008) 
showed that pure no-overbidding Nash equilibria are guaranteed to exist for SPIB auctions when all prodution functions are fractionally subadditive. Several additional papers continued to study this model, analyzing its "price of anarchy", which measures the efficiency level guaranteed by any no-overbidding Nash equilibrium, when it exists, relative to the optimal efficiency level. For example, Bhawalkar and Roughgarden (2011) showed that when buyers' valuations are subadditive, a no-overbidding equilibrium, when it exists, always guarantees an efficiency level that is at least half of the maximum possible. The literature also studies the "price of stability" of various models, which is the efficiency that the best Nash equilibrium outcome guarantees relative to the optimal efficiency.

The purpose of this note is to point at the connection of no-overbidding Nash equilibria of the SPIB auction game to JS-stable outcomes. The notion of no-overbidding we use here is a relaxation of the one used in Christodoulou et al. (2008) and Bhawalkar and Roughgarden (2011):

Definition 6. A NE of the SPIB auction is weakly-no-overbidding if the bids of each bidder on the subset of items allocated to her does not exceed her value for these items.

Nash equilibria that are weakly no-overbidding clearly contain all Nash equilibria with no-overbidding. Our main result is as follows:

Theorem 2. There exists a pure Nash equilibrium with weak-no-overbidding in the SPIB auction of a labor market if and only if there exists a JS-stable outcome in this market. Furthermore, the assignments of workers to firms in the Nash equilibria outcomes are identical to the assignments in the JS-stable outcomes (though salaries need not be identical).

In other words, this theorem ties existence of JS-stable outcomes to existence of pure NE with weak no-overbidding. It also asserts that workers' assignments in both types of outcomes are identical, while salaries may differ. In particular, in the construction used in our proof, the Nash equilibrium salaries are generally lower than the JS-stable salaries (for the same assignments).

Proof. (Theorem 2 Let $\vec{p}$ be a NE with weak no-overbidding of the SPIB auction and let then $A^{n}=D^{n}(\vec{p})$ denote the set of workers assigned to firm $n$ in that equilibrium. Set a new salary for each worker $m \in M$ to be $s_{m}=p_{m}^{n(m)}$, i.e., the salary proposed by the firm that receives this worker in the equilibrium. We now argue that $(A, s)$ is a JS-stable outcome. To see this note that as the equilibrium had the weak no-overbidding property, $v^{n}\left(A^{n}\right) \geq \sum_{m \in A^{n}} s_{m}$, hence this outcome is IR. The fact that $\vec{p}$ is a NE implies that, for any firm $n$ and $C \subset M \backslash A^{n}$, $v^{n}\left(C \mid A^{n}\right) \leq \sum_{m \in C} p_{m}^{n(m)}=\sum_{m \in C} s_{m}$. Thus, there does not exist a blocking coalition for $(A, s)$, and the claim follows.

As for the other direction let $(A, s)$ be a JS-stable outcome. Consider the following bidding strategy in the SPIB auction:

$$
p_{m}^{n}= \begin{cases}s_{m} & \text { if } m \in A^{n} \\ 0 & \text { if } m \notin A^{n}\end{cases}
$$

We claim that $\vec{p}$ is a NE with weak no-overbidding. Note that $\vec{p}$ induces an allocation where firm $n$ wins $A^{n}$, and pays zero. JS-stability implies $v^{n}\left(A^{n}\right) \geq \sum_{m \in A^{n}} s_{m}$, hence $p^{n}$ satisfies weak no-overbidding.

To verify that this is a Nash equilibrium, fix a firm $n$, and suppose towards a contradiction that there exists a strictly profitable deviation from $p^{n}$ for firm $n$. Suppose that the firm receives some 
set of workers $X$ in this deviation. Since $n$ pays a salary of zero for each worker $m \in A^{n}$ we can assume without loss of generality that $A^{n} \subset X$. To win any $m \in X \backslash A^{n}$, firm $n$ must submit a salary larger than $s_{m}$, therefore it will pay a salary of $s_{m}$ to $m$. Since $\left(n, X \backslash A^{n}\right)$ is not a blocking coalition, we have $v^{n}\left(X \backslash A^{n} \mid A^{n}\right) \leq \sum_{m \in X \backslash A^{n}} s_{m}$. Thus, $X$ does not strictly increase $n$ 's utility, a contradiction. The claim follows.

On top of the non-cooperative strategic reasoning to the emergence of JS-stable assignments, the formal connection between the two models coupled with Theorem 1 from our previous paper applies to questions of existence and efficiency of Nash equilibria outcomes studied in the literature on simultaneous single-item auctions. In particular, the combination of Theorem 1 and Theorem 2 immediately implies:

Corollary 1. The price of anarchy of any second-price item-bidding auction is 2, regardless of the class of production functions. In other words, if a weakly no-overbidding equilibrium exists, its efficiency level is at least half of the maximum attainable by any allocation.

Note that this result does not assume anything about the production functions, while all other results in this literature need to assume something about the production functions.

Corollary 2. When all bidders' valuations are in AFS, a weakly-no-overbidding efficient Nash equilibrium is guaranteed to exist. Thus, the price of stability of any second-price item-bidding auction with valuations in AFS is 1. Furthermore, AFS is the maximal class of valuations that guarantees these properties.

\section{References}

Bhawalkar, K. and Roughgarden, T. (2011). Welfare guarantees for combinatorial auctions with item bidding. In Proceedings of the 22nd Symposium On Discrete Algorithms (SODA), pages 700-709.

Bikhchandani, S. (1999). Auctions of heterogeneous objects. Games and Economic Behavior, $\underline{26(2): 193-220 .}$.

Christodoulou, G., Kovács, A., and Schapira, M. (2008). Bayesian combinatorial auctions. In Proceedings of the 35th International Colloquium on Automata, Languages, and Programming (ICALP), pages $820-832$.

Fu, H., Kleinberg, R., and Lavi, R. (2012). Conditional equilibrium outcomes via ascending price processes with applications to combinatorial auctions with item bidding. In The 13th ACM Conference on Electronic Commerce (EC'12), page 586.

Fu, H., Kleinberg, R., Lavi, R., and Smorodinsky, R. (2016). Job security, stability and production efficiency. Theoretical Economics. In press.

Gul, F. and Stacchetti, E. (1999). Walrasian equilibrium with gross substitutes. Journal of Economic Theory, 87:95-124.

Kelso, A. S. and Crawford, V. (1982). Job matching, coalition formation and gross substitutes. Econometrica, 50(6):1483-1504. 
Lehmann, B., Lehmann, D., and Nisan, N. (2006). Combinatorial auctions with decreasing marginal utilities. Games and Economic Behavior, 55(2):270-296. 